\title{
CIRUGÍA RENAL CONSERVADORA DE NEFRONAS COMO TRATAMIENTO DE RESCATE TRAS FRACASO DE LA TERMOABLACIÓN MEDIANTE RADIOFRECUENCIA EN PACIENTE MONORRENO CON ADENOCARCINOMA RENAL.
}

\author{
Enrique Fernández Rosado, Javier Sánchez Rodríguez-Losada, Venancio Chantada Abal \\ y José Manuel Rois Soto'.
}

Urólogos. Anatomía Patológica'. USP-Policlínico Santa Teresa. La Coruña. España.

\begin{abstract}
Resumen.- OBJETIVO: Descripción de la técnica de la termoablación renal mediante radiofrecuencia (TARF). Presentación de una cirugía renal conservadora de nefronas como tratamiento exitoso de rescate tras fracaso de la TARF de un adenocarcinoma renal en un paciente monorreno quirúrgico.
\end{abstract}

MÉTODOS: Un adenocarcinoma renal izquierdo parcialmente exofítico en un paciente monoreno es tratado mediante TARF. En el seguimiento de la misma se observa fracaso de dicha técnica al comprobar la existencia de una zona central necrótica rodeada de una zona periférica captadora de contraste. Se decide cirugía renal conservadora como técnica de rescate.

RESULTADOS: Extirpación quirúrgica del tumor y tejido renal próximo con margen de seguridad, sin clampaje
Enrique Fernández Rosado C/ Ronda de Outeiro, 133 - 2 Izda. 15007 La Coruña (España) efrosado@ozu.es

Trabajo recibido: 20 de enero 2006 del pediculo renal. Aposición de tejido graso y material hemostático sintético en el lecho quirúrgico Estudio anatomopatológico: adenocarcinoma renal con cambios secundarios a necrosis en su zona central. Seguimiento postcirugía de 18 meses: nódulo graso renal con pequeños tractos fibrosos en su interior en la zona previamente ocupada por el tumor. Sin evidencia de ninguna zona captante de contraste en el TAC. Función renal normal (Crp 0,7 mg/dl, Urea $24 \mathrm{mg} / \mathrm{dl}$ ).

CONCLUSIONES: La TARF es una técnica relativamente nueva. Su eficacia oncológica depende en gran medida de la selección adecuada de los casos a tratar. Uno de sus principales problemas radica en el hecho de conseguir una zona de ablación tisular suficiente como para destruir totalmente el tumor. En caso de fracaso de la técnica, la cirugía renal (conservadora de nefronas o no) parece la alternativa mas recomendada.

Palabras clave: Ablación mediante radiofrecuencia. Adenocarcinoma renal. Cirugía conservadora.

Summary.- OBJECTIVES: To describe the technique of renal radiofrequency (RF) thermal ablation. Case report of a successful nephron-sparing surgery after failure of the RF thermal ablation of a renal adenocarcinoma in a patient with a single kidney.

METHODS: A patient presenting with a renal adenocarcinoma in a single left kidney was treated by RF thermal ablation. The failure of the technique was patent on follow-up after demonstration of a central area of necrosis surrounded by a peripheral contrast enhancing area. Nephron sparing surgery was indicated as salvage procedure. 
RESULTS: Surgical excision of the tumor with a safety margin, without renal pedicle clamping was undertaken. Fat tissue and hemostatic synthetic material were placed in the surgical bed. Pathology report: renal adenocarcinoma with changes secondary to central necrosis. Twenty-four month postoperative follow-up: fatty renal nodule with small fibrous tracts inside in the area of the tumor. No evidence of contrast enhancing areas. Normal renal function $(\mathrm{s} C r 0.7 \mathrm{mg} / \mathrm{dl}$, urea $24 \mathrm{mg} / \mathrm{d}$ ).

CONCLUSIONS: Radiofrequency thermal ablation is a relatively new technique. Its oncological efficacy greatly depends on appropriate case selection. One of its main caveats is achieving an area of tissue ablation enough to completely destroy the tumor. When the technique fails, renal surgery (nephron sparing or not) seems to be the most recommended alternative.

Keywords: Radiofrequency ablation. Renal adenocarcinoma. Conservative surgery.

\section{INTRODUCCIÓN}

Desde mediados del siglo pasado, el diagnóstico de los adenocarcinomas renales ha ido creciendo de modo importante debido a las mejoras tecnológicas y a la generalización de los estudios de imagen abdominales. Así, el número de casos que se detectan en la actualidad de modo incidental en pacientes asintomáticos se aproxima al 50\% (1). Ello ha generado interés en técnicas que no requieren la nefrectomía radical ("gold standar" del tratamiento de los adenocarcinomas renales). La nefrectomía parcial - la cirugía renal conservadora de nefronas, y más recientemente la criocirugía renal y la termoablación mediante radiofrecuencia (TARF) tienen su lugar en pacientes adecuadamente seleccionados.

El uso del calor inducido por una corriente eléctrica que atraviesa un tejido a una frecuencia determinada fue empleado por primera vez para el tratamiento de tumores cerebrales. Debido a que esta corriente eléctrica que generaba ondas electroquirúrgicas tenía una frecuencia similar a las ondas de radio, se las denominó ondas de radiofrecuencia. Posteriormente se trataron tumores hepáticos, se investigó en su uso en tumores renales (2) y en 1999 se publicó el primer caso de un adenocarcinoma renal tratado mediante TARF percutánea guiada por ecografía (3).

En la TARF la corriente generada se transmite hasta la punta no aislada del electrodo activo introducido en la zona de ablación. La energía allí liberada induce una agitación iónica, que da lugar a la aparición de fricción y calor localizado alrededor del electrodo. Este calor se concentra en una zona pequeña de tejido, generándose altas temperaturas. Cuando se llega a los $50^{\circ} \mathrm{C}$ durante más de 3-6 minutos, acontece la desnaturalización intracelular de las proteínas y la fusión de las bicapas lipídicas, con la aparición de necrosis de coagulación ("kill zone"). El empleo de varios electrodos unidos (grupos de electrodos) aumenta el volumen de la necrosis de coagulación. Tanto la desecación como el "chamuscado" del tejido alrededor de la punta del electrodo limitan la propagación de la corriente de radiofrecuencia (RF). Existen métodos para disminuir este fenómeno: la inyección de suero salino intraparenquimatosa durante el proceso de la TARF (lo cual aumenta la conductividad y la superficie eficaz de tratamiento); los electrodos con sistemas internos de enfriamiento lque enfrían la punta del electrodo, disminuyendo el calentamiento de los tejidos próximos y el fenómeno de desecación-chamuscado); la aplicación pulsada de energía de RF (que disipa el calor en la punta del electrodo periódicamente, lo que impide también la desecación-chamuscado); el control de la temperatura del electrodo (que permite controlar la zona de ablación) (4).

Es fundamental en la TARF la adecuada selección de los casos a tratar. Generalmente son pacientes no candidatos a cirugía, con tumores pequeños (generalmente inferiores a $3-4 \mathrm{~cm}$.), de localización accesible (mediante la vía percutánea (5), laparoscópica o cirugía abierta) y periféricos. Tumores de localización renal central y los situados próximos a vasos sanguíneos grandes son de difícil y arriesgado tratamiento mediante esta técnica, debido a que el flujo continuo de sangre enfría constantemente el tejido, impidiendo su adecuado y eficaz calentamiento (efecto denominado "sumidero de calor"), además del riesgo de lesión térmica de los vasos.

En un futuro, con nuevas innovaciones y mejorías técnicas, se podrá mejorar esta técnica, al incrementar el volumen de tumor a tratar y su completa ablación. Aun así, como ocurre con todas las técnicas de reciente aparición, la evolución oncológica de los pacientes tratados con TARF a largo plazo es incierta, debido principalmente al desconocimiento de la evolución de volúmenes tumorales mínimos viables en los bordes de las zonas tratadas (6).

Presentamos un caso clínico único con iconografía completa, en donde tras el fracaso de la TARF, correctamente indicada en un paciente especialmente seleccionado, se realizó una cirugía renal conservadora de nefronas como tratamiento exitoso de rescate de un adenocarcinoma renal en un paciente monorreno quirúrgico. 


\section{MATERIAL Y MÉTODOS}

Paciente de 66 años, monorreno izquierdo quirúrgico de la infancia tras tuberculosis renal. También fue tratado mediante LEOC, tras la colocación de stent doble "J" por anuria y fracaso renal agudo, de litiasis renal y ureteral izquierda. En una consulta de rutina (asintomático), se le detecta ecográficamente la presencia, en el riñón izquierdo con hipertrofia compensadora $(14 \mathrm{~cm}$.), de una lesión sólida exofítica de unos $2 \mathrm{~cm}$. a nivel de la zona media renal (Figura 1A). Tras la realización de un TAC con contraste se demostró que dicha lesión parcialmente exofítica captadora de contraste, aunque con centro probablemente necrótico, correspondía posiblemente a un adenocarcinoma renal incidental de $2,3 \mathrm{~cm}$. de diámetro máximo (Figura 1B).

Debido a las especiales características del paciente y del tumor, desde un inicio se procuró ofertarle un tratamiento conservador: nefrectomía parcial conservadora de nefronas por cirugía abierta o mediante laparoscopia; termoablación mediante radiofrecuencia (TARF); crioterapia. Finalmente, explicados al paciente los pros y contras de cada tratamiento, y la posibilidad de perdida de función renal y de precisar diálisis al ser monoreno, el paciente tras informarse ampliamente de las técnicas y consultar la opinión de otros urólogos, decidió el tratamiento mediante TARF.

Se le realizó TARF durante un periodo de 19 minutos, alcanzando una temperatura final de $53,5^{\circ} \mathrm{C}$.
El tamaño tumoral previo al tratamiento era de 3,7 $\mathrm{cm}$. de diámetro máximo. La interposición del bazo dificultó la punción de la parte más superior del tumor (Figura 2). El TAC post-TARF al mes del tratamiento, aprecia la existencia de un área central necrótica, persistiendo una captación periférica de contraste indicando claramente actividad a dicho nivel, con un diámetro máximo de 4,2 cm. (Figura 3).

Con el diagnostico de persistencia del adenocarcinoma renal tras fracaso de la TARF y dificultad para el tratamiento en una nueva sesión de la zona mas alta del tumor, se planteó como medida terapéutica de rescate la cirugía conservadora.

\section{RESULTADOS}

Se realizó finalmente una tumorectomía a cielo abierto con un margen de seguridad de medio centímetro en toda su periferia intrarenal. No se realizó clampaje del pedicuro renal con el fin de no dañar más parénquima. La hemorragia durante la cirugía fue escasa y se realizó cuidadosa hemostasia. El defecto en el parénquima tras la extirpación del tumor fue rellenado con grasa y material sintético hemostático (Figura 4). Tras 5 días de postoperatorio sin complicaciones fue dado de alta con función renal normal.

El estudio anatomopatológico mostró la presencia de un carcinoma de células renales tipo
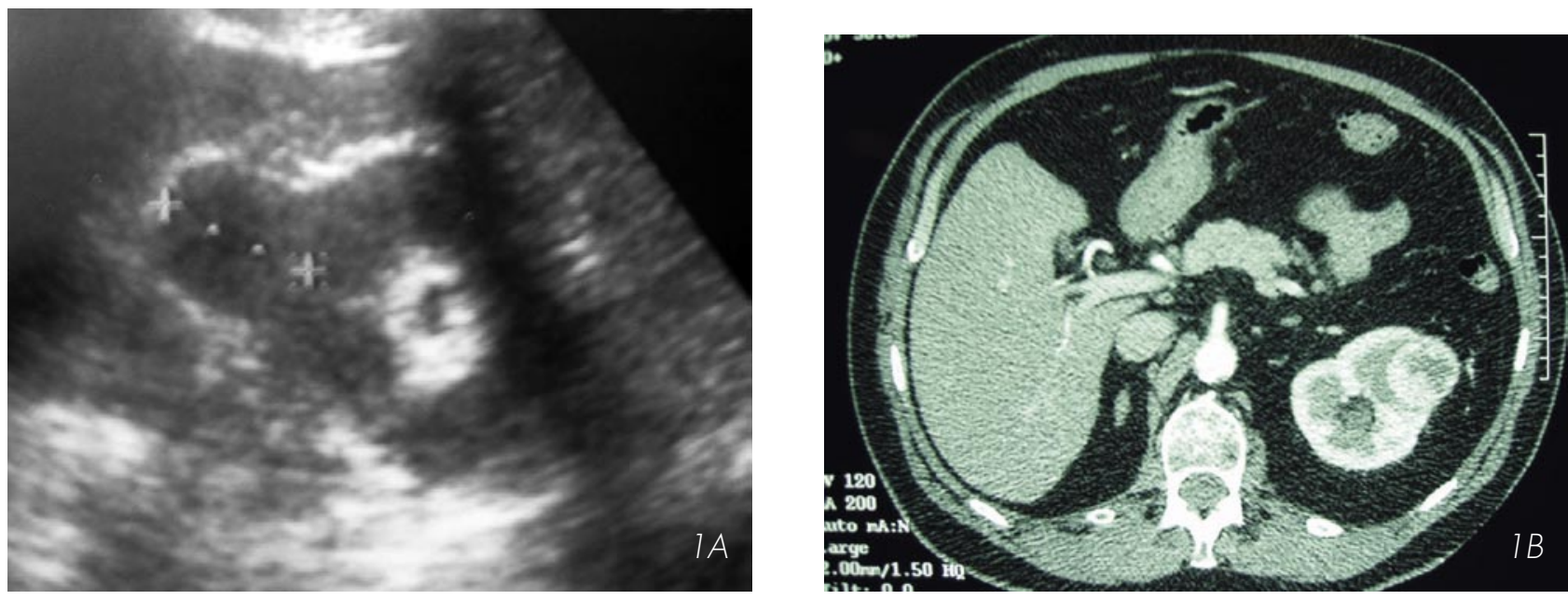

FIGURA IA y 1B. Diagnóstico ecográfico incidental (la) en riñón izquierdo con hipertrofia compensadora (paciente monorreno quirúrgico por atrofia renal derecha post-tuberculosal, de una lesión sólida exofítica de unos 2 cm. a nivel de la zona media renal. Tras la realización de un TAC con contraste (1 b) se demostró que dicha lesión parcialmente exofítica captadora de contraste, aunque con centro probablemente necrótico, correspondía posiblemente a un adenocarcinoma renal incidental de $2,3 \mathrm{~cm}$. de diámetro máximo. 


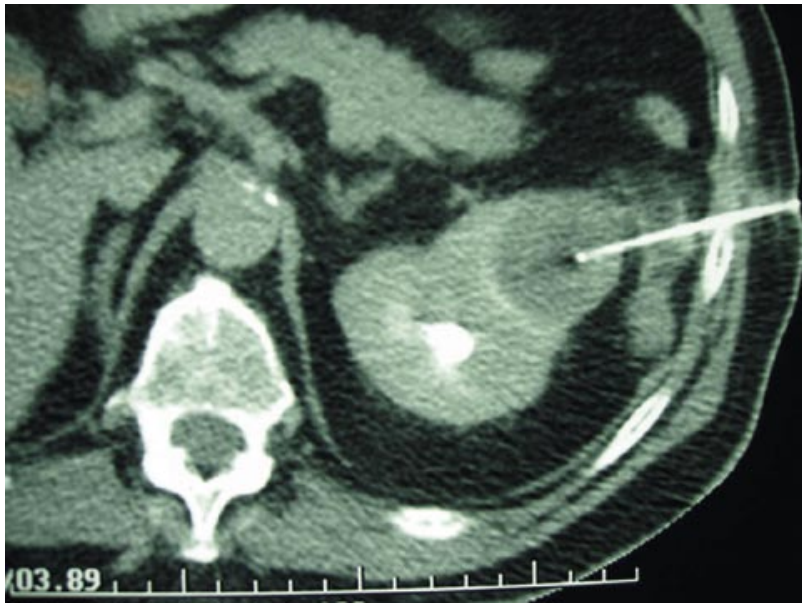

FIGURA 2. Termoablación renal mediante radiofrecuencia, alcanzando durante un periodo de 19 minutos una temperatura final de $53,5^{\circ} \mathrm{C}$.

células claras, grado nuclear de Furhman I/IV, con patrón de crecimiento sólido, de diámetro máximo de $3 \mathrm{~cm}$. (Figura 5A). Aproximadamente en $1 / 3$ de la masa tumoral, en su zona más central, se evidencia necrosis de coagulación en relación con el tratamiento térmico previo, con fibronecrosis y ausencia total

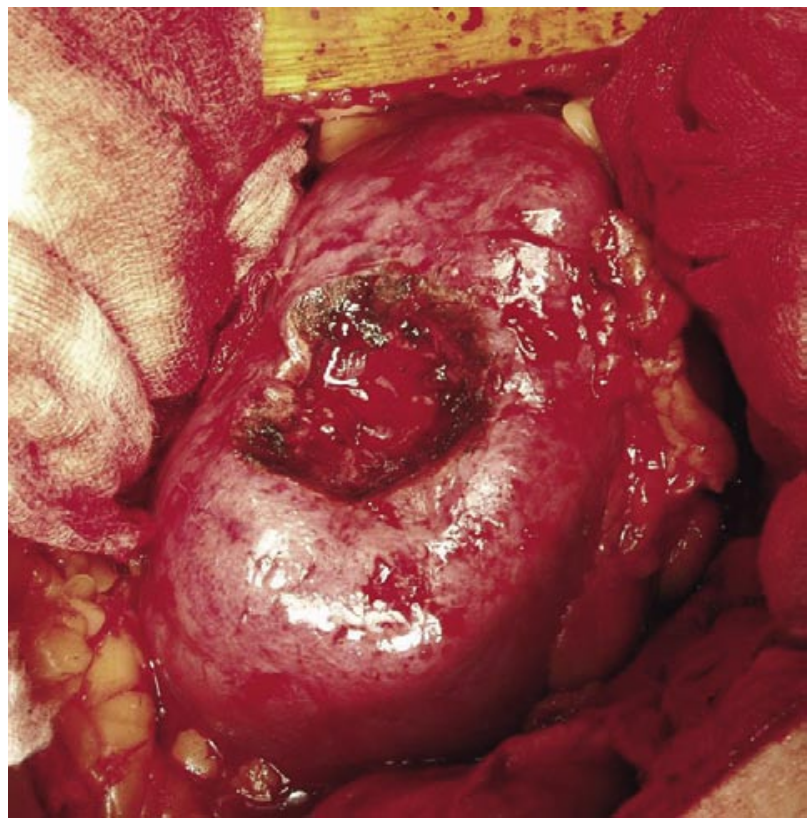

FIGURA 4. Cirugía renal conservadora de nefronas sin clampaje del pedículo renal: tumorectomía con margen de resección de seguridad de $0,5 \mathrm{~cm}$. en la zona intrarenal del tumor.

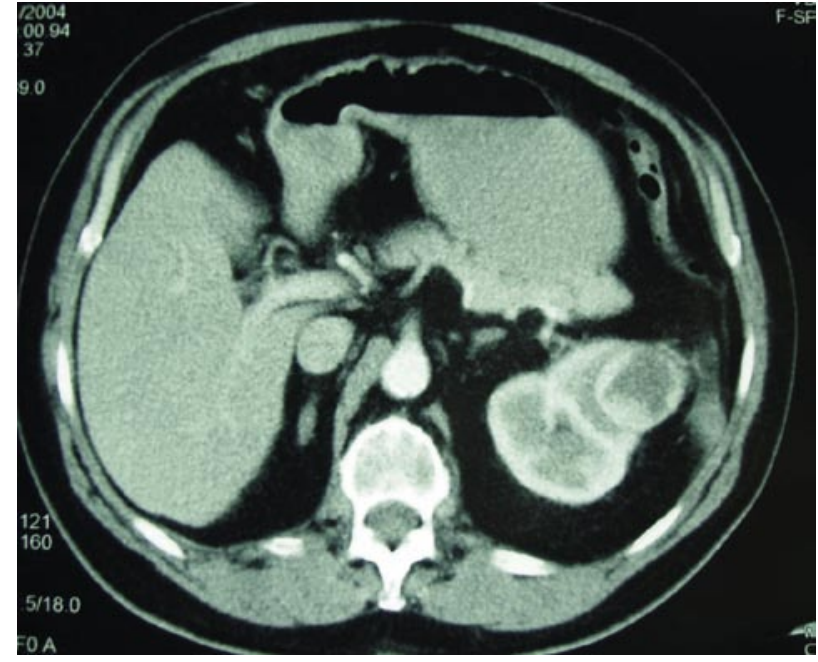

FIGURA 3. TAC de control post-tratamiento (1 mes). Área central necrótica con captación periférica de contraste, evidenciando el fracaso de la sesión de radiofrecuencia y la existencia de tejido tumoral viable.

de células tumorales (Figura 5B). El tejido tumoral viable es periférico a esta zona de necrosis central y con una clara zona de "transición" (Figura 5C), y se encuentra rodeado de parénquima renal sin alteraciones con un ancho entre $0,1-0,3 \mathrm{~cm}$.

El seguimiento actual del paciente es de 18 meses tras la cirugía conservadora de rescate. Tanto las ecografías como los TAC de seguimiento muestran la presencia de un nódulo graso renal en la localización previa del tumor, con pequeños tractos fibrosos en su interior y sin evidencia de ninguna zona captante de contraste (Figura 6). La función renal es normal con unos valores de Crp 0,7 mg/dl y Urea 24 $\mathrm{mg} / \mathrm{dl}$.

\section{CONCLUSIONES}

La TARF es una técnica relativamente reciente -primer caso publicado en 1999 (3)-, válida para la ablación de tejido tumoral renal (lo destruye mediante necrosis por coagulación), y relativamente sencilla de aplicar (pudiendo realizarse de modo percutáneo y de manera ambulatoria bajo anestesia general o sedación). En la actualidad sus indicaciones y su uso tienden a generalizarse, empleándose con éxito en recidivas tumorales (7), en tumores en injertos renales trasplantados (7), como terapia paliativa alternativa a la embolización renal (8) y otros.

Progresivamente están apareciendo publicadas series de algunos centros con un número de casos tratados importantes (9-11). Dichas series y las 


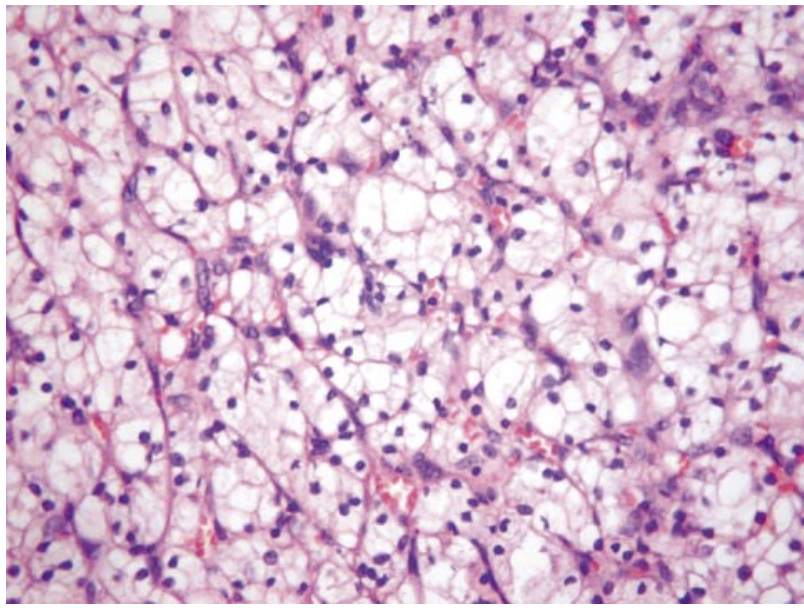

FIGURA 5A. Estudio anatomopatológico de la zona mas periférica del tumor: carcinoma de células renales tipo células claras, patrón sólido, grado nuclear de Furhman I/IV (100x). Zona de fracaso de la ablación mediante radiofrecuencia.

que ya se pueden encontrar publicadas carecen de una revisión y una evolución a largo plazo en la actualidad (actualmente la serie con mayor seguimiento medio no lega a los 3 años), por lo que se desconoce realmente la efectividad de la TARF para desbancar a la cirugía conservadora en estos casos especiales.

Su eficacia oncológica depende en gran medida de la selección adecuada de los casos a

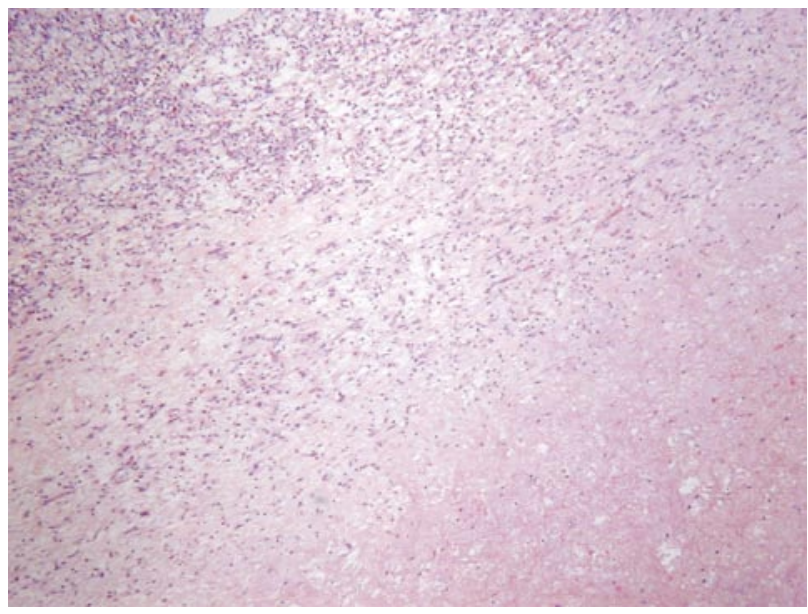

FIGURA 5C. Estudio anatomopatológico de la zona de "transición" del tumor, entre la zona central (necrótica) y la periférica (celular): células tumorales de citoplasma claro (extremo superior izquierdo) y zona central tumoral con fibronecrosis y ausencia total de células tumorales (extremo inferior derecho).

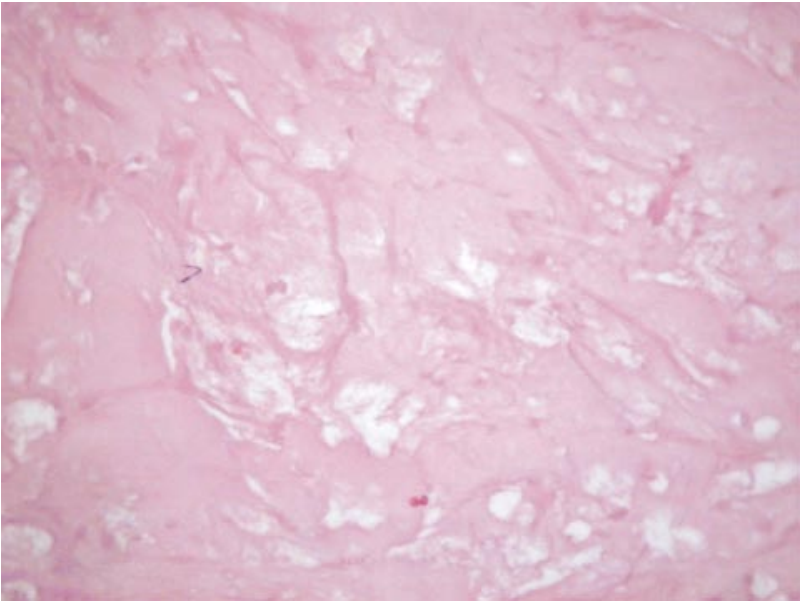

FIGURA 5B. Estudio anatomopatológico de la zona central del tumor ("kill zone"): fibronecrosis de coagulación sin evidencia de células tumorales. Zona de destrucción celular total por la radiofrecuencia.

tratar. Uno de los principales problemas radica en el hecho de conseguir una zona de ablación tisular suficiente como para destruir totalmente el tumor. A pesar de la mejoría técnica, la repetición de sesiones, la refrigeración de los electrodos o el empleo de energía pulsátil, el volumen de tejido a tratar es una de las principales limitaciones de este tratamiento. Pero, sin embargo, y tal y como se demuestra en el estudio anatomopatológico de este caso, las zonas

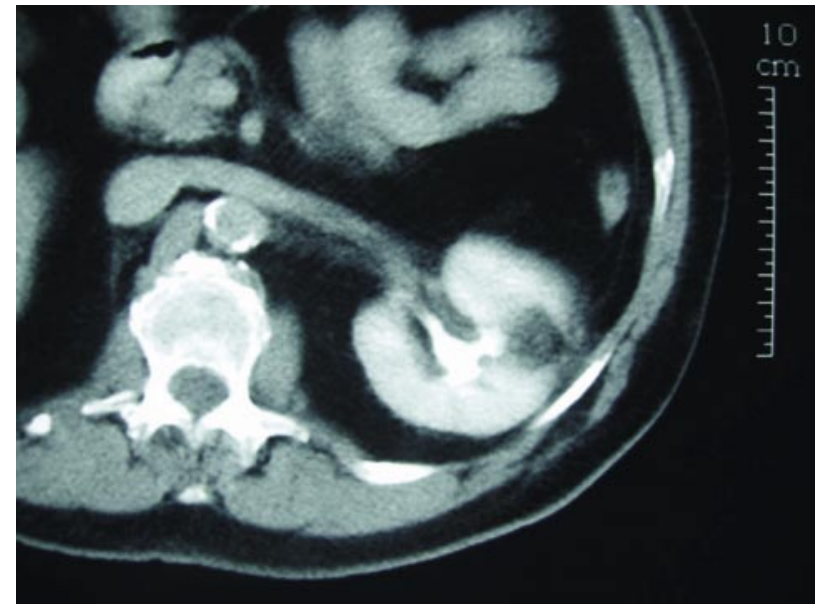

FIGURA 6. TAC de control a los 18 meses postcirugía: nódulo graso renal con tractos fibrosos en su interior y sin captación de contraste en la localización previa del tumor. 
afectadas por la energía térmica (zona central del tumor) muestran una necrosis de coagulación total sin evidencia de células tumorales, lo que demuestra la alta eficacia de la TARF para destruir por completo el tejido tratado. El problema está, obviamente, en el tejido que queda sin tratar $y$ en su verdadera viabilidad oncológica.

En caso de fracaso de una sesión de TARF, es posible someter al paciente a nuevas sesiones con la finalidad de destruir completamente el tejido tumoral. La cirugía renal de rescate (conservadora de nefronas $\circ$ no) parece la alternativa mas recomendada frente al fracaso final de sesiones consecutivas o ante la imposibilidad de tratar zonas debido a su posición o interposición de otros órganos o tejidos.

\section{BIBLIOGRAFÍA Y LECTURAS RECOMENDADAS ( lectura fundamental)}

*1. FERNÁNDEZ ROSADO, E.; SUÁREZ PASCUAL, G.; BLANCO DIEZ, A. y cols.: "Clinical-pathological study of incidental renal cell carcinoma". Arch. Esp. Urol., 58: 635, 2005.

*2. ZLOTTA, A.R.; WILDSCHUTZ, T.; RAVIV, G. y cols.: "Radiofrequency interstitial tumor ablation (RITA) is a possible new modality for treatment of renal cancer: ex vivo and in vivo experience". J. Endourol., 1: 251, 1997.

3. McGOVERN, F.J.; WORD, B.J.; GOLDBERG, S.N. y cols.: "Radio frecuency ablation of renal cell carcinoma via image guided needle electrodes”. J. Urol., 161: 599, 1999.
**4. GAZELLE, G.S.; GOLDBERG, S.N.; SOLBIATI, L. y cols.: "Tumor ablation with radiofrecuency energy". Radiology, 217: 633, 2000.

**5. OGAN, K.; JACOMIDES, L.; DOLMATCH, B.L. y cols.: "Percutaneus radio-frecuency ablation of renal tumors: technique, limitations and morbidity". Urology, 60: 954, 2000.

*6. RENDON, R.A.; KACHURA, J.R.; SWEET, J.M. y cols.: "The uncertainty of radio frecuency treatment of renal cell carcinoma: findings at immediate and delayed nephrectomy". J. Urol., 167: 1587, 2002.

7. GOEMAN, L.; JONIAU, S.; OYEN, R. y cols.: "Percutaneous ultrasound-guided radiofrequency ablation of recurrent renal cell carcinoma in renal allograft after partial nephrectomy". Urology, 67: 199, 2006.

8. MARCHAL, F.; BRUNAUD, L.; BAZIN, C. y cols.: "Radiofrequency ablation in palliative supportive care: Early clinical experience". Oncol. Rep., 15: 495, 2006.

**9. McDOUGAL, W.S.; GERVAIS, D.A.; McGOVERN, F.J. y cols.: "Long-term followup of patients with renal cell carcinoma treated with radio frequency ablation with curative intent". J. Urol., 174: 61, 2005.

**10. GERVAIS, D.A.; McGOVERN, F.J.; ARELLANO, R.S. y cols.: "Radiofrequency ablation of renal cell carcinoma: part 1, Indications, results, and role in patient management over a 6-year period and ablation of 100 tumors". Am. J. Roentgenol.,185: 64, 2005.

**11. GERVAIS, D.A.; McGOVERN, F.J.; ARELLANO, R.S. y cols.: "Radiofrequency ablation of renal cell carcinoma: part 2, Lessons learned with ablation of 100 tumors". Am. J. Roentgenol., 185: 72, 2005. 